\title{
Familial secondhand smoke: Tobacco use and adoption of smoke-free home and car rules among US parents
}

\author{
Israel T. Agaku ${ }^{1}$, Tina N. Tsafa ${ }^{2}$, Katerina Nikitara ${ }^{3}$, Satomi Odani ${ }^{4}$
}

\author{
AFFILIATION \\ 1 Department of Oral Health Policy and Epidemiology, Harvard School \\ of Dental Medicine, Boston, United States \\ 2 Benue State University, Makurdi, Nigeria \\ 3 School of Medicine, University of Crete, Heraklion, Crete \\ 4 Cancer Control Center, Osaka International Cancer Institute, Osaka, \\ Japan
}

CORRESPONDENCE TO

Israel T. Agaku. Department of Oral Health Policy and Epidemiology,
Harvard School of Dental Medicine, 188 Longwood Avenue, Boston, Massachusetts 02115, United States. E-mail: iagaku@post.harvard.edu

\section{KEYWORDS}

secondhand smoke, smoke-free home, tobacco products

Received: 4 January 2021, Revised: 7 July 2021, Accepted: 8 July 2021

\begin{abstract}
INTRODUCTION Secondhand smoke (SHS) causes disease and death. We assessed US parents' tobacco use and their attitudes towards smoking within private environments where children might be present.

METHODS A national sample of 44626 parents collectively reporting 83782 children aged 0-17 years was analyzed from the 2014-2015 Tobacco Use Supplement to the Current Population Survey. Unit of analyses was both parents and children. Among parents, we assessed tobacco use prevalence, smoke-free home rule adoption, and opposition to smoking in cars. Logistic regression was used to measure associations between smoke-free home rule adoption and parents' cigarette smoking initiation (never smokers); quit attempts (current smokers); and sustained cessation (former smokers). Population counts of children living with a smoking parent were extrapolated from sampling weights.
\end{abstract}

RESULTS Of parents, $14.3 \%$ currently smoked combustible tobacco; approximately 9.7 million children lived with a smoking parent. While most parents opposed smoking in cars with children (95.0\%), significantly fewer were opposed when a child was not specified as being present in the car (75.4\%). Overall, $91.3 \%$ of parents had smoke-free home rules; this percentage was highest among parents of infants/ toddlers (92.3\%) and lowest among parents of teens aged $14-17$ years $(89.0 \%$; $p<0.05)$. Presence of smoke-free home rules was associated negatively with smoking initiation among never smokers $(\mathrm{AOR}=0.21)$ and positively with quit attempts among current smokers $(\mathrm{AOR}=1.59)$ and sustained quitting among former smokers (AOR=1.67) (all p <0.05). CONCLUSIONS Parental smoking can expose children to SHS. Pediatricians can educate parents on the dangers of smoking around children, and the benefits of quitting.

impaired lung function, and middle ear disease ${ }^{3}$. SHS exposure has also been shown to be associated with poor academic performance among children ${ }^{4,5}$. Addressing SHS exposure in the home and car, therefore, aligns closely with public health priorities on early brain development and overall adolescent development, with further implications for public health programs and policies.

Besides these health-related considerations, tobacco use by parents can have a negative effect on social norms by renormalizing tobacco use. Youths whose parents smoke are more likely to smoke themselves and to start smoking at an earlier age ${ }^{6}$. Furthermore, children who are exposed to secondhand aerosol from electronic cigarettes have increased curiosity and susceptibility to using both 
e-cigarettes and traditional cigarettes compared to those unexposed $^{7}$. Proximal social contacts such as family and friends are frequently cited among youth for various tobacco-related behaviors, such as reason for starting certain tobacco product use, and usual source of accessing tobacco products $^{8}$. Presence of tobacco products around the house can provide visual and olfactory cues that could potentially lead to relapse among those attempting to quit ${ }^{9}$. The impact of smoking in the home is not only limited to those within the immediate confines of that household given that SHS can infiltrate into neighboring living units and other shared areas; an estimated one-third of US multi-unit housing residents experience SHS infiltration in the units each year ${ }^{10}$.

Progress has been made in recent years in protecting youth from SHS exposure in different public and private settings where children are typically present. Nine US states, Guam, the Northern Mariana Islands and Puerto Rico have prohibited smoking in cars with a child passenger ${ }^{11}$. In 2016, the U.S. Department of Housing and Urban Development finalized a rule that prohibits smoking in public housing, protecting the nation's 2 million public housing residents, including 0.76 million children, from SHS in their homes ${ }^{12}$. As of November 2017, 27 states, the District of Columbia, and over 900 local municipalities had implemented comprehensive smoke-free laws ${ }^{13}$; nearly $60 \%$ of US residents are currently covered by comprehensive smoke-free laws at the state or local level. However, millions of Americans are still exposed to SHS, and disparities in exposure exist across subpopulations ${ }^{8}$.

There is paucity of recent, nationally representative data on tobacco use among US parents, as well as parental adoption of smoke-free rules in the home and car, and the benefits of such voluntary policies on smokingrelated outcomes. In addition, while previous research has estimated the number of US school-going children in grades 6-12 exposed to SHS within the home ${ }^{14,15}$, there is paucity of data on potential exposures among children of all ages between 0-17 years regardless of their schoolenrollment status (i.e. both school-going and non-schoolgoing children). To fill these gaps in knowledge, we analyzed nationally representative data from the 2014-2015 Tobacco Use Supplement to the Current Population Survey (TUS-CPS), using both parents and their children as units of analyses. The objectives of the present study are to: 1) describe the tobacco product use patterns among US parents, their attitudes towards smoking within the home or a car where children might be present; 2 ) investigate the potential benefits of having smoke-free rules within private environments on parental smoking behaviors; and 3) estimate the number of US children who are exposed to SHS within private environments.

\section{METHODS}

\section{Data source}

TUS-CPS is a survey of the civilian, non-institutionalized
US adult population aged $\geq 18$ years conducted as part of the U.S. Census Bureau's Current Population Survey. The sampling frame for the Current Population Survey is 119 million US households from the civilian non-institutionalized population. Households are randomly selected by the Bureau of the Census on the basis of mailing addresses to represent the nation as a whole, individual states, and other specified areas. One to three individuals were randomly selected for self-interview from each household according to the size of the household. The 2014-2015 TUS-CPS had a total of 163920 self-respondents; yielding an overall response rate of $54.2 \%$. Interviews were conducted in July 2014 , January 2015, and May 2015, four to six months apart. TUS-CPS provides estimates critical to understanding the burden of tobacco use in the US and the extent to which it is concentrated in particular subgroups. The large sample size of TUS-CPS particularly equips it to provide smaller subsample estimates (e.g. parents) with greater precision.

In this study, our target population was parents of children under 18 years of age related by birth, marriage, or adoption who reported the presence of their own children in their household. A total of 44626 parents reported a combined number of 83782 own children aged 0-17 years. For each type of family unit identified in the CPS, the count of children aged 0-17 years was limited to single (never married) children.

\section{Measures}

Home and car smoke-free rules and opinions among parents Participants were asked: 'Which statement best describes the rules about smoking INSIDE YOUR HOME? (Note: "Home" is where you live. "Rules" include any unwritten rules and pertain to all people whether or not they reside in the home or are visitors, workmen, etc. Smoking includes cigars regular and hookah as well as cigarettes.)'. A response of 'No one is allowed to smoke anywhere INSIDE YOUR HOME' (vs 'Smoking is allowed in some places or at sometimes INSIDE YOUR HOME'; or 'Smoking is permitted anywhere INSIDE YOUR HOME') was classified as having a complete smokefree home rule.

Attitudes towards smoking inside a car were assessed under two scenarios: 1) Without specifying the presence of a child passenger, 'Inside a car, when there are other people present, do you THINK that smoking SHOULD ... ?'; and 2) Specifically indicating that a child was present, 'IF children are present inside the car, do you think that smoking SHOULD ...?'. Response options to both questions were: 'Always be allowed', 'Be allowed under some conditions', and 'Never be allowed'. The last response was classified as completely opposing smoking in cars for the specific scenario assessed.

\section{Tobacco use and sociodemographic characteristics among} parents

Six tobacco product types were assessed in TUS-CPS: 
cigarettes; cigars/cigarillos/filtered little cigars; smokeless tobacco products; regular pipes; water pipes; and e-cigarettes. Current users were persons who reported ever use ( $\geq 100$ cigarettes in lifetime, or $\geq 1$ time in lifetime for all other products) and reported the use of the respective products 'every day' or 'some days' at the time of survey. Any tobacco product use was defined as use of any of the six assessed tobacco product types, and any combustible tobacco product use was defined as using any of cigarettes, cigars/cigarillos/filtered little cigars, regular pipes, or water pipes. We further classified respondents based on exclusive use patterns, as non-users of any tobacco product; users of only combustible tobacco products; users of only smokeless tobacco products; users of only e-cigarettes; and users of a combination of tobacco products.

Household characteristics reported by parents included number of own children; age of youngest/only child (0-2; $3-5$; 6-13; or 14-17 years); and family structure (married, only one parent present; married, both parents present; widowed/divorced/separated; never married). Other sociodemographic characteristics included parents' race/ ethnicity, educational attainment, U.S. Census region, metropolitan status, annual household income, veteran status (whether having served in the US military or not), and status of combustible tobacco use (never/former/current [some days or every day]).

\section{Smoking-related outcomes}

Smoking-related outcomes were assessed among current cigarette smokers (smoked $\geq 100$ cigarettes in lifetime and smoke now), former cigarette smokers (smoked $\geq 100$ cigarettes in lifetime but no longer smoke), and never cigarette smokers (smoked $<100$ cigarettes, or never, in lifetime). Among current cigarette smokers, we assessed past-year quit attempt, as well as intentions to quit smoking in the next 30 days and 6 months, respectively. Among former smokers, we assessed sustained quitting, defined as having stopped smoking for 6 months or longer. Among never smokers, we assessed past 5-year cigarette smoking initiation; numerator was persons who started smoking within the past 5 years; denominator was those who started smoking within the past 5 years as well as those who had never smoked cigarettes in their lifetime.

\section{Analyses}

The unit of analyses included both parents (primary unit) and children aged 0-17 years (secondary unit). Among parents, we computed tobacco use prevalence, and the percentage who reported adopting voluntary home smokefree rules and opposing smoking in a car with or without children specified as being present. Within-group differences were assessed using Pearson's chi-squared tests. Logistic regression models were fitted to measure the relationship between adoption of smoke-free home rules and various smoking-related outcomes, adjusting for number of children, age of youngest/only child, family structure, and parental age, sex, race/ethnicity, non-cigarette tobacco product use, annual household income, and education level. To check potential correlations between covariates, we computed variance inflation factors (VIFs) for each of the independent variables in the models, and confirmed all VIFs were $<10$.

With children aged 0-17 years as the unit of analysis, we estimated the total count of those who were potentially exposed to secondhand smoke by virtue of living with a parent that used tobacco products. Probability weights were used to extrapolate population counts of children in different types of households based on tobacco usage. To ensure that estimates generated were nationally representative, TUS-CPS self-response weights derived from the Census Bureau were applied. All analyses were performed with R.V.3.5.1. using the 'survey' statistical package. Statistical significance was set at $\mathrm{p}<0.05$.

\section{RESULTS}

Of all US adults who completed the 2014-2015 TUS-CPS, $28.3 \%$ were parents; total number of own children reported ranged from 1 to 12 , nationwide. Overall, $41.6 \%$ of parents had one child, $37.8 \%$ had two children, and $20.6 \%$ had $\geq 3$ children. The age of the only/youngest child was $0-2$ years for $16.3 \%$ of parents, $3-5$ years for $21.2 \%$ of parents, $6-13$ years for $43.8 \%$ of parents, and $14-17$ years for $18.7 \%$ of parents.

\section{Parental tobacco use behavior}

Prevalence of current any tobacco use among parents was $16.3 \%$; prevalence of current any combustible tobacco smoking was $14.3 \%$ (Table 1). By specific tobacco product type, prevalence was as follows: cigarettes (13.1\%); cigars $(1.7 \%)$; hookahs/water pipes $(0.3 \%)$; regular pipes $(0.1)$; smokeless tobacco products (1.7\%); and e-cigarettes (2.4\%).

Any current tobacco use prevalence among parents in two-parent families $(12.9 \%)$ was about half that in all other family structures, including those married, but only one spouse present (23.8\%); those never married (25.7\%); and those widowed, divorced, or separated (25.1\%). Parents with one child only had the highest prevalence of current any tobacco product use (17.5\%) compared to those with two (15.4\%) or $\geq 3$ children (15.6\%). By US census region, parental tobacco use prevalence was highest in the Midwest $(21.5 \%)$ and lowest in the West (11.1\%). Prevalence was almost two-fold higher among parents residing in nonmetropolitan areas $(27.7 \%)$ than those in metropolitan areas (14.6\%); as well as among veterans (26.7\%) compared to non-veterans (14.6\%). By education level, prevalence of current any tobacco use was highest among those with a high school diploma (24.6\%), and lowest among those with $\geq$ college (6.5\%). Similarly, prevalence was highest among those with annual household income (US\$) of $<20 \mathrm{~K}(25.9 \%)$ and lowest among those earning $\geq 100 \mathrm{~K}(8.8 \%)$. By race/ ethnicity, prevalence was highest among non-Hispanic 
Table 1. National estimates of current tobacco use by parents, by selected sociodemographic characteristics, Tobacco Use Supplement to the Current Population Survey, 2014-2015

\begin{tabular}{|c|c|c|c|c|c|c|c|c|}
\hline & $\begin{array}{l}\text { Any tobacco } \\
\text { product }\end{array}$ & $\begin{array}{c}\text { Any } \\
\text { combustible } \\
\text { tobacco product } \\
\%(95 \% \mathrm{CI})\end{array}$ & $\begin{array}{l}\text { Cigarette } \\
\%(95 \% \mathrm{CI})\end{array}$ & $\begin{array}{c}\text { Cigar/cigarillo/ } \\
\text { little cigar } \\
\%(95 \% \mathrm{CI})\end{array}$ & $\begin{array}{c}\text { Pipe } \\
\%(95 \% \mathrm{CI})\end{array}$ & $\begin{array}{l}\text { Hookah } \\
\%(95 \% \text { CI })\end{array}$ & $\begin{array}{c}\begin{array}{c}\text { Smokeless } \\
\text { tobacco }\end{array} \\
\%(95 \% \mathrm{CI})\end{array}$ & $\begin{array}{c}\begin{array}{c}\text { Electronic } \\
\text { cigarette }\end{array} \\
\%(95 \% \mathrm{CI})\end{array}$ \\
\hline Overall & $16.3(15.9-16.7)$ & $14.3(13.9-14.7)$ & $13.1(12.7-13.5)$ & $1.7(1.6-1.9)$ & $0.1(0.1-0.2)$ & $0.3(0.2-0.4)$ & $1.7(1.6-1.9)$ & $2.4(2.2-2.5)$ \\
\hline \multicolumn{9}{|l|}{ Family structure } \\
\hline Married, spouse present & $12.9(12.5-13.3)$ & $10.8(10.4-11.2)$ & $9.5(9.1-9.8)$ & $1.7(1.5-1.9)$ & $0.1(0.1-0.2)$ & $0.2(0.1-0.2)$ & $1.9(1.8-2.1)$ & $1.8(1.6-2)$ \\
\hline Married, spouse absent & $23.8(19.3-28.3)$ & $21.6(17.2-25.9)$ & $20.9(16.6-25.2)$ & & - & - & - & $3.2(1.4-5.1)$ \\
\hline Widowed/divorced/separated & $25.1(23.8-26.4)$ & $22.8(21.6-24.1)$ & $22.3(21.1-23.6)$ & $1.5(1.1-1.8)$ & - & $0.5(0.3-0.8)$ & $1.5(1.2-1.9)$ & $4.2(3.6-4.8)$ \\
\hline Never married & $25.7(24.3-27)$ & $24.2(22.9-25.6)$ & $23.1(21.8-24.4)$ & $2.1(1.6-2.5)$ & - & $0.8(0.5-1.1)$ & $1(0.7-1.3)$ & $3.5(3-4.1)$ \\
\hline \multicolumn{9}{|l|}{ Number of children } \\
\hline 1 & $17.5(16.8-18.1)$ & $15.5(14.9-16.1)$ & $14.2(13.6-14.8)$ & $1.8(1.6-2.1)$ & $0.1(0.1-0.2)$ & $0.4(0.3-0.5)$ & $1.7(1.5-1.9)$ & $2.6(2.4-2.9)$ \\
\hline 2 & $15.4(14.8-16.1)$ & $13.3(12.7-13.9)$ & $12(11.4-12.6)$ & $1.7(1.5-2)$ & $0.2(0.1-0.2)$ & $0.3(0.2-0.4)$ & $1.8(1.6-2)$ & $2.2(1.9-2.5)$ \\
\hline$\geq 3$ & $15.6(14.7-16.5)$ & $13.8(13-14.6)$ & $12.8(12-13.6)$ & $1.5(1.2-1.8)$ & - & $0.2(0.1-0.3)$ & $1.7(1.4-2)$ & $2.2(1.8-2.5)$ \\
\hline \multicolumn{9}{|l|}{ Census region } \\
\hline Northeast & $15.1(14-16.1)$ & $14(13-15)$ & $12.8(11.9-13.8)$ & $1.8(1.4-2.2)$ & - & - & $0.7(0.5-0.9)$ & $1.8(1.4-2.1)$ \\
\hline Midwest & $21.5(20.5-22.5)$ & $18.5(17.6-19.4)$ & $17(16.2-17.9)$ & $2(1.7-2.4)$ & $0.2(0.1-0.3)$ & $0.4(0.2-0.6)$ & $2.8(2.4-3.2)$ & $3(2.6-3.4)$ \\
\hline South & $17.5(16.8-18.2)$ & $15.2(14.6-15.9)$ & $14.1(13.4-14.7)$ & $1.7(1.5-2)$ & $0.1(0.1-0.2)$ & $0.3(0.2-0.4)$ & $2(1.8-2.2)$ & $2.7(2.4-3)$ \\
\hline West & $11.1(10.5-11.8)$ & $9.7(9-10.3)$ & $8.6(8-9.2)$ & $1.4(1.2-1.7)$ & $0.2(0.1-0.2)$ & $0.3(0.2-0.5)$ & $1.2(1-1.4)$ & $1.8(1.5-2.1)$ \\
\hline \multicolumn{9}{|l|}{ Metro status } \\
\hline Metro & $14.6(14.2-15)$ & $12.9(12.5-13.3)$ & $11.7(11.3-12.1)$ & $1.7(1.6-1.9)$ & $0.1(0.1-0.2)$ & $0.3(0.2-0.4)$ & $1.3(1.1-1.4)$ & $2.2(2-2.4)$ \\
\hline Non-metro & 27.7 (26.5-29) & $23.3(22.2-24.5)$ & $22.4(21.3-23.6)$ & $1.8(1.4-2.1)$ & $0.2(0.1-0.4)$ & $0.3(0.1-0.4)$ & $4.8(4.1-5.4)$ & $3.6(3.1-4.1)$ \\
\hline \multicolumn{9}{|l|}{ Veteran status } \\
\hline Veteran & $26.7(24.5-28.9)$ & $21.7(19.7-23.8)$ & $18.7(16.7-20.7)$ & $4.5(3.5-5.6)$ & - & - & $4.8(3.8-5.8)$ & $5.3(4.1-6.4)$ \\
\hline Non-veteran & $15.8(15.4-16.3)$ & $14(13.6-14.3)$ & $12.8(12.5-13.2)$ & $1.6(1.4-1.7)$ & $0.1(0.1-0.2)$ & $0.3(0.2-0.4)$ & $1.6(1.5-1.7)$ & $2.2(2.1-2.4)$ \\
\hline \multicolumn{9}{|l|}{ Education level } \\
\hline$<$ High school & $21.1(19.7-22.5)$ & $19.9(18.6-21.3)$ & $19.6(18.2-20.9)$ & $1.6(1.1-2)$ & - & $0.6(0.2-0.9)$ & $1.2(0.9-1.6)$ & $2.7(2.1-3.3)$ \\
\hline High school & $24.6(23.6-25.6)$ & $21.9(21-22.8)$ & $20.8(19.9-21.7)$ & $2(1.7-2.4)$ & $0.1(0.1-0.2)$ & $0.3(0.1-0.4)$ & $2.7(2.3-3)$ & $3.2(2.8-3.6)$ \\
\hline Some college & $20(19.2-20.8)$ & $17.3(16.5-18)$ & $15.8(15.1-16.6)$ & $1.9(1.6-2.2)$ & $0.2(0.1-0.2)$ & $0.4(0.3-0.5)$ & $2.1(1.8-2.3)$ & $3.5(3.2-3.9)$ \\
\hline College or higher & $6.5(6.1-7)$ & $5.3(4.9-5.7)$ & $3.9(3.5-4.2)$ & $1.5(1.2-1.7)$ & $0.1(0.1-0.2)$ & $0.2(0.1-0.3)$ & $1(0.9-1.2)$ & $0.8(0.7-1)$ \\
\hline
\end{tabular}


Table 1. Continued

\begin{tabular}{|c|c|c|c|c|c|c|c|c|}
\hline & $\begin{array}{l}\text { Any tobacco } \\
\text { product } \\
\%(95 \% \mathrm{CI})\end{array}$ & $\begin{array}{c}\text { Any } \\
\text { combustible } \\
\text { tobacco product } \\
\%(95 \% \mathrm{CI})\end{array}$ & $\begin{array}{l}\text { Cigarette } \\
\%(95 \% \mathrm{CI})\end{array}$ & $\begin{array}{c}\text { Cigar/cigarillo/ } \\
\text { little cigar } \\
\%(95 \% \mathrm{CI})\end{array}$ & $\begin{array}{c}\text { Pipe } \\
\%(95 \% \mathrm{CI})\end{array}$ & $\begin{array}{c}\text { Hookah } \\
\%(95 \% \mathrm{CI})\end{array}$ & $\begin{array}{c}\begin{array}{c}\text { Smokeless } \\
\text { tobacco }\end{array} \\
\%(95 \% \mathrm{CI})\end{array}$ & $\begin{array}{c}\begin{array}{c}\text { Electronic } \\
\text { cigarette }\end{array} \\
\%(95 \% \mathrm{CI})\end{array}$ \\
\hline \multicolumn{9}{|l|}{ Income (US\$) } \\
\hline 0-19999 & $25.9(24.6-27.2)$ & $24.6(23.3-25.9)$ & $23.8(22.6-25.1)$ & $1.8(1.4-2.2)$ & - & $0.6(0.4-0.9)$ & $1(0.7-1.3)$ & $3.7(3.2-4.3)$ \\
\hline 20000-49999 & $20.7(19.9-21.6)$ & $18.5(17.7-19.3)$ & $17.7(16.9-18.5)$ & $1.6(1.3-1.9)$ & $0.2(0.1-0.3)$ & $0.5(0.3-0.6)$ & $1.8(1.5-2)$ & $3.2(2.9-3.6)$ \\
\hline 50000-99999 & $14.6(13.9-15.2)$ & $12.3(11.7-12.9)$ & $11.2(10.6-11.8)$ & $1.5(1.2-1.7)$ & $0.1(0.1-0.2)$ & $0.2(0.1-0.3)$ & $2.2(1.9-2.4)$ & $2(1.7-2.3)$ \\
\hline$\geq 100000$ & $8.8(8.2-9.4)$ & $6.8(6.3-7.3)$ & $4.8(4.4-5.2)$ & $2.1(1.8-2.4)$ & $0.1(0.1-0.2)$ & $0.1(0-0.2)$ & $1.7(1.4-1.9)$ & $1.2(1-1.4)$ \\
\hline \multicolumn{9}{|c|}{ Race/ethnicity } \\
\hline White & $20.3(19.8-20.9)$ & $17.2(16.7-17.8)$ & $15.8(15.3-16.3)$ & $1.9(1.7-2.1)$ & $0.2(0.1-0.2)$ & $0.3(0.2-0.3)$ & $2.7(2.5-3)$ & $3.3(3-3.5)$ \\
\hline Black & $14.9(13.6-16.1)$ & $14.3(13.1-15.5)$ & $12.5(11.3-13.6)$ & $2.8(2.2-3.5)$ & - & $0.7(0.3-1)$ & - & $1.1(0.7-1.5)$ \\
\hline Asian & $6.3(5.2-7.4)$ & $5.9(4.9-7)$ & $5.5(4.5-6.5)$ & - & - & - & - & $0.4(0.2-0.7)$ \\
\hline Hispanic & $8.7(8-9.5)$ & $8.3(7.6-9)$ & $7.9(7.2-8.6)$ & $0.9(0.7-1.2)$ & - & $0.3(0.1-0.4)$ & $0.3(0.1-0.4)$ & $0.9(0.7-1.2)$ \\
\hline Other & $24.5(21.5-27.6)$ & $21.9(18.9-24.8)$ & $20.3(17.4-23.1)$ & $2(0.9-3.1)$ & - & - & $2(1.1-2.9)$ & $5.2(3.4-7)$ \\
\hline
\end{tabular}

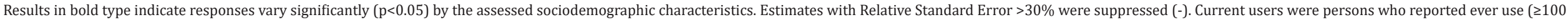

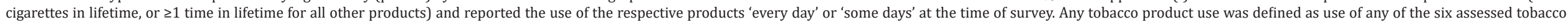
product types, and any combustible tobacco product use was defined as using any of cigarettes, cigars/cigarillos/filtered little cigars, regular pipes, or water pipes. 
Whites (20.3\%) and lowest among Hispanics (8.7\%).

By exclusive use patterns, 83.6\% (56.5 million) of parents used no form of tobacco at all; $12.3 \%$ (8.3 million) used combustible tobacco products exclusively, $1.3 \%(857,000)$ used smokeless tobacco products exclusively, 0.7\% $(0.5$ million) used e-cigarettes exclusively, and 2.0\% (1.36 million) used a combination of products.

An estimated 56.50 million US children aged 0-17 years lived with a parent that did not use any tobacco product; 11.04 million lived with a parent that used any form of tobacco; while 9.7 million children lived with a parent that smoked a combustible tobacco product. The number of children by exclusive tobacco product usage by parents is shown in Table 2.

\section{Parental rules and opinions on smoking in the home and in a car}

Overall, $91.3 \%$ of parents reported adopting complete smoke-free home rules (Table 3). Prevalence was: highest among two-parent families living together (93.5\%) and lowest among those never married (85.5\%); highest among those with two children (92.6\%) and lowest among those with one child (90.3\%); highest in households with an infant/toddler (92.3\%) and lowest among households where the oldest child was 14-17 years old (89.0\%). Relatively lower prevalence of adoption of home smoke-free rules was seen among the following parent groups: military veterans (89.8\%); earning <20 K/annum (82.2\%); daily smokers $(63.8 \%)$, and residents of non-metropolitan areas (85.9\%). By exclusive use patterns, adoption of complete smoke-free home rules was as follows: non-tobacco users (95.1\%), combustible-only users (69.7\%); smokeless tobacco-only users (92.7\%); e-cigarette-only users (89.3\%); and users of a combination of products (69.1\%).

Almost all parents (95.1\%), including those who smoked daily $(83.3 \%)$ or some days (93.5\%), were opposed to smoking in a car with a child present. However, significantly fewer parents were opposed to smoking in a car when a child was not specified present (75.4\%). Only $43.2 \%$ of parents who smoked daily, and $59.2 \%$ of those who smoked on some days, opposed smoking in a car when a child was not specified present. By exclusive use patterns, percentage

Table 2. Estimated number of children based on status of complete smoke-free home rule, Tobacco Use Supplement to the Current Population Survey, 2014-2015

\begin{tabular}{|c|c|c|c|c|c|c|c|}
\hline \multirow{2}{*}{$\begin{array}{l}\text { Current } \\
\text { tobacco } \\
\text { product use }\end{array}$} & \multicolumn{4}{|c|}{ Parents: Weighted percentages, $\%$ (95\% CI) } & \multicolumn{3}{|c|}{ Children: Weighted counts } \\
\hline & $\begin{array}{c}\text { Sample } \\
\text { size }\end{array}$ & $\begin{array}{l}\text { Complete } \\
\text { smoke-free } \\
\text { home rule }\end{array}$ & $\begin{array}{c}\text { Oppose } \\
\text { smoking in cars } \\
\text { in general }\end{array}$ & $\begin{array}{l}\text { Oppose } \\
\text { smoking in } \\
\text { cars when } \\
\text { children } \\
\text { specified } \\
\text { present }\end{array}$ & $\begin{array}{l}\text { Extrapolated } \\
\text { number of } \\
\text { children aged } \\
0-17 \text { years, } \\
\text { total }\end{array}$ & $\begin{array}{c}\text { Extrapolated } \\
\text { number } \\
\text { of children } \\
\text { aged 0-17 } \\
\text { years, } \\
\text { households } \\
\text { with } \\
\text { complete } \\
\text { smoke-free } \\
\text { home rules }\end{array}$ & $\begin{array}{l}\text { Extrapolated } \\
\text { number of } \\
\text { children aged } \\
\text { 0-17 years, } \\
\text { households } \\
\text { with no } \\
\text { complete } \\
\text { smoke-free } \\
\text { home rules }\end{array}$ \\
\hline Overall & 44626 & $91.3(91.1-91.4)$ & $75.4(75.1-75.7)$ & $95.1(94.9-95.3)$ & 68387270 & 61499510 & 6887760 \\
\hline $\begin{array}{l}\text { No tobacco } \\
\text { product }\end{array}$ & 36403 & 95.1 (94.9-95.3) & $80.6(80.2-81.1)$ & 96.7 (96.5-96.9) & 56500690 & 53371510 & 3129180 \\
\hline $\begin{array}{l}\text { Any tobacco } \\
\text { product }\end{array}$ & 7674 & $72.3(72.1-73.5)$ & $48.9(47.5-40.3)$ & $87.1(86.8-87.3)$ & 11035105 & 7922598 & 3112507 \\
\hline \multicolumn{8}{|l|}{$\begin{array}{l}\text { Any } \\
\text { combustible } \\
\text { tobacco } \\
\text { product }\end{array}$} \\
\hline $\begin{array}{l}\text { Combustible } \\
\text { only }\end{array}$ & 5747 & $69.7(68.3-71.1)$ & $49.5(47.8-51.1)$ & $86.6(85.6-87.5)$ & 8321001 & 5760208 & 2560793 \\
\hline $\begin{array}{l}\text { Smokeless } \\
\text { tobacco only }\end{array}$ & 651 & $92.7(90.3-95.1)$ & $61.2(56.4-66.1)$ & $92.5(89.9-895.0)$ & 857233 & 792939 & 64294 \\
\hline $\begin{array}{l}\text { E-cigarettes } \\
\text { only }\end{array}$ & 326 & $89.3(85.9-93.8)$ & $47.2(40.4-53.9)$ & $93.0(90.0-96.1)$ & 499911 & 439398 & 60513 \\
\hline Combination & 950 & $69.1(65.6-72.7)$ & $38.2(34.4-42.0)$ & $84.6(81.9-87.4)$ & 1356959 & 930052 & 426907 \\
\hline
\end{tabular}


Table 3. Proportion of US parents that prohibit smoking in their homes and oppose smoking within cars, Tobacco Use Supplement to the Current Population Survey, 2014-2015

\begin{tabular}{|c|c|c|c|c|}
\hline & $\mathbf{n}$ & Home & Car in general & Car with children \\
\hline Overall & 44626 & $91.3(91.0-91.6)$ & $75.4(74.9-75.9)$ & $95.1(94.9-95.4)$ \\
\hline \multicolumn{5}{|l|}{ Sex } \\
\hline Male & 17893 & $92.1(91.6-92.6)$ & $72.7(71.9-73.4)$ & 94.5 (94.1-94.9) \\
\hline Female & 26733 & $90.8(90.4-91.2)$ & $77.4(76.8-78.0)$ & 95.6 (95.3-95.9) \\
\hline \multicolumn{5}{|l|}{ Race/ethnicity } \\
\hline White, non-Hispanic & 29302 & $91.3(90.9-91.7)$ & $69.0(68.4-69.6)$ & $93.3(93.0-93.7)$ \\
\hline Black, non-Hispanic & 4370 & $86.8(85.7-88.0)$ & $79.3(77.9-80.8)$ & $96.6(96.0-97.3)$ \\
\hline Asian, non-Hispanic & 2326 & $95.9(90.5-96.8)$ & $88.0(86.4-89.5)$ & 98.5 (97.9-99.0) \\
\hline Hispanic & 7302 & $93.0(92.3-93.7)$ & $87.1(86.2-88.0)$ & $98.0(97.6-98.4)$ \\
\hline Other, non-Hispanic & 1326 & $86.8(84.2-89.3)$ & $69.7(66.3-73.1)$ & $95.2(93.7-96.7)$ \\
\hline \multicolumn{5}{|l|}{ Education level } \\
\hline$<$ High school & 4233 & $84.9(83.7-86.2)$ & $81.0(79.6-82.4)$ & $95.2(94.5-95.9)$ \\
\hline High school & 10784 & $86.7(8.06-87.5)$ & $74.5(73.5-75.5)$ & $94.8(94.3-95.3)$ \\
\hline Some college & 12949 & $90.7(90.1-91.3)$ & $73.5(72.6-74.4)$ & $94.9(94.5-95.4)$ \\
\hline College or higher & 16660 & $96.8(96.5-97.2)$ & 75.8 (75.1-76.6) & 95.5 (95.1-95.8) \\
\hline \multicolumn{5}{|l|}{ Income (US\$) } \\
\hline 0-19999 & 5939 & $82.2(81.1-83.4)$ & $73.8(72.5-75.2)$ & $93.7(9.03-94.5)$ \\
\hline 20000-49999 & 11989 & $88.2(87.5-88.9)$ & $76.3(75.4-77.2)$ & $95.1(94.7-95.6)$ \\
\hline 50000-99999 & 14356 & $93.9(93.4-94.4)$ & $75.4(74.5-76.2)$ & $95.6(95.2-96.0)$ \\
\hline$\geq 100000$ & 12342 & 96.5 (96.1-96.9) & $75.4(74.5-76.3)$ & $95.3(94.9-95.8)$ \\
\hline \multicolumn{5}{|l|}{ Family structure } \\
\hline Married, spouse present & 32666 & $93.5(93.1-93.8)$ & $76.6(76.1-77.1)$ & $95.5(95.2-95.8)$ \\
\hline Married, spouse absent & 481 & $87.5(83.8-91.2)$ & $76.9(72.3-81.5)$ & $94.9(92.2-97.5)$ \\
\hline Widowed/divorced/separated & 5724 & $85.9(84.8-86.9)$ & $71.4(70.1-72.8)$ & $92.8(92.0-93.5)$ \\
\hline Never married & 5755 & $85.5(84.4-86.6)$ & $72.6(71.2-74.0)$ & $95.2(94.5-95.8)$ \\
\hline \multicolumn{5}{|l|}{ Number of children } \\
\hline 1 & 18574 & $90.3(89.8-90.8)$ & $75.1(74.4-75.8)$ & $95.1(94.7-95.5)$ \\
\hline 2 & 16853 & $92.6(92.2-93.1)$ & $75.1(74.3-75.9)$ & $95.0(94.7-95.4)$ \\
\hline$\geq 3$ & 9199 & $91.1(90.4-91.8)$ & $76.7(75.6-77.7)$ & $95.3(94.8-95.9)$ \\
\hline \multicolumn{5}{|c|}{ Age of youngest/only child (years) } \\
\hline $0-2$ & 6465 & $92.3(91.5-93.1)$ & $75.3(74.0-76.6)$ & 96.1 (95.5-96.7) \\
\hline $3-5$ & 8407 & $91.3(90.5-92.0)$ & $76.1(75.0-77.2)$ & $96.0(95.5-96.4)$ \\
\hline $6-13$ & 17386 & $91.4(90.9-91.9)$ & $75.6(74.9-76.3)$ & $94.3(93.9-94.7)$ \\
\hline $14-17$ & 7437 & $89.0(88.1-89.8)$ & $76.1(74.9-77.2)$ & 94.0 (93.4-94.6) \\
\hline \multicolumn{5}{|l|}{ Region } \\
\hline Northeast & 7176 & $90.9(90.1-91.8)$ & $76.2(75.0-77.4)$ & 96.1 (95.6-96.7) \\
\hline Midwest & 9801 & $89.2(88.4-89.9)$ & $66.6(65.5-67.7)$ & $92.5(91.8-93.1)$ \\
\hline South & 15685 & $90.3(89.8-90.8)$ & 77.1 (76.4-77.9) & $94.9(94.5-95.3)$ \\
\hline West & 11964 & $95.0(94.5-95.5)$ & $79.7(78.8-80.6)$ & $97.0(96.6-97.3)$ \\
\hline
\end{tabular}


Table 3. Continued

\begin{tabular}{|c|c|c|c|c|}
\hline & $\mathbf{n}$ & Home & Car in general & Car with children \\
\hline \multicolumn{5}{|l|}{ Metropolitan status } \\
\hline Metro & 35317 & $92.1(91.8-92.5)$ & $76.3(75.8-76.8)$ & $95.5(95.2-95.7)$ \\
\hline Non-metro & 8745 & $85.9(85.0-86.9)$ & $69.2(67.9-70.5)$ & $92.9(92.2-93.6)$ \\
\hline \multicolumn{5}{|l|}{ Veteran } \\
\hline Yes & 2141 & $91.3(88.2-91.3)$ & $67.7(65.4-70.1)$ & $93.0(91.7-94.3)$ \\
\hline No & 42485 & 91.7 (91.1-91.7) & $75.8(75.3-76.2)$ & $95.2(95.0-95.5)$ \\
\hline \multicolumn{5}{|c|}{ Combustible tobacco use status } \\
\hline Never user & 27673 & $95.2(94.9-95.5)$ & $84.4(83.9-84.9)$ & $97.3(97.0-97.5)$ \\
\hline Former user & 9753 & $94.3(93.7-94.9)$ & $66.6(65.5-67.8)$ & $94.7(94.2-95.2)$ \\
\hline Current user - some days & 1885 & $83.7(81.7-85.8)$ & $58.8(56.1-61.5)$ & $93.4(92.0-94.7)$ \\
\hline Current user - every day & 4807 & $63.8(62.2-65.4)$ & $43.4(41.7-45.1)$ & $83.4(82.1-84.6)$ \\
\hline
\end{tabular}

For each type of family unit identified in the CPS, the count of own children under 18 years old was limited to single (never married) children related to the respondent by birth, marriage, or adoption. Results in bold type indicate responses vary significantly $(\mathrm{p}<0.05)$ by the assessed sociodemographic characteristics. Combustible tobacco product use was defined as using any of cigarettes, cigars/cigarillos/filtered little cigars, regular pipes, or water pipes.

opposing smoking in a car in general versus when children were specified as present, respectively, was as follows: nontobacco users ( $80.6 \%$ vs $96.7 \%$ ), combustible-only users (49.5\% vs $86.6 \%)$; smokeless tobacco-only users $(61.2 \%$ vs $92.5 \%)$, e-cigarette-only users (47.2\% vs $93.0 \%)$, and users of a combination of products $(38.2 \%$ vs $84.6 \%)$.

\section{Association between smoke-free home policies and smoking-related outcomes}

Smoke-free home rules had beneficial effects on smoking- related behaviors and attitudes among US parents. Among never cigarette smokers, odds of initiating cigarette smoking were higher among those with than without complete smoke-free home rules (AOR=0.21; 95\%CI: 0.13-0.33) (Figure 1). Among those who were current cigarette smokers, odds were higher among those with than without complete smoke-free home rules of making a past-year quit attempt (AOR=1.59; 95\% CI: 1.37-1.84), intending to quit in the next 30 days (AOR=1.72; 95\%CI: 1.43-2.08), or in the next 6 months (AOR=1.55; 95\% CI: 1.34-1.80). Among former

Figure 1. Adjusted odds ratios of smoking-related outcomes among parents with voluntary smoke-free home rules compared to those without voluntary home rules, Tobacco Use Supplement to the Current Population Survey, 2014-2015

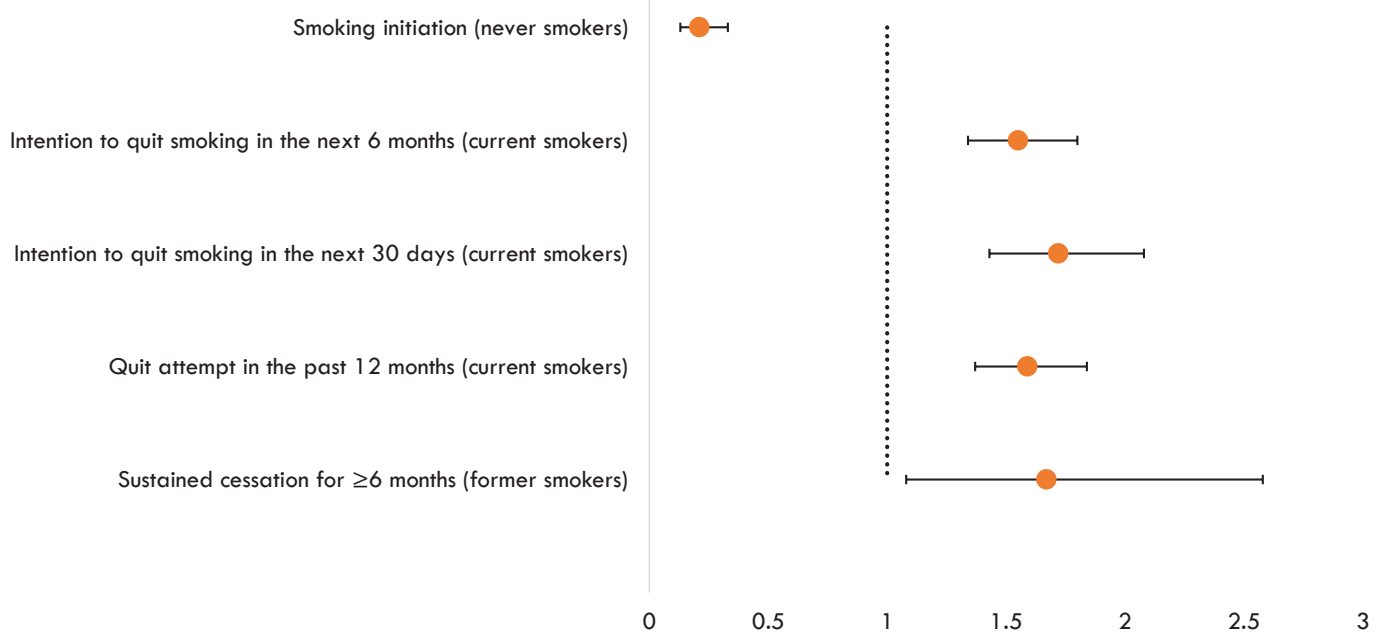

Logistic regression models were adjusted for number of children, age of youngest/only child, family structure, and parental age, sex, race/ethnicity, non-cigarette tobacco product use, and education level. 
smokers, the odds of reporting sustained quitting were also higher among those with than without complete smoke-free home rules (AOR=1.67; 95\% CI: 1.08-2.58).

\section{DISCUSSION}

One in four US parents used a tobacco product, and 11.04 million children lived with a parent that used any form of tobacco. The overwhelming majority of US parents reported having complete smoke-free home rules (91.3\%) and were also opposed to smoking in a car with children present $(95.1 \%)$. However, significantly fewer parents (75.4\%) were opposed to smoking in a car when a child was not specified present. SHS can adhere to surfaces and possibly expose youth to tobacco smoke even after active smoking has ceased (i.e. thirdhand smoke) ${ }^{16,17}$. Despite the high support for smoke-free home/car rules by parents, a recent study indicates that $29.0 \%$ (7.5 million) of US middle and high school students were exposed to SHS within their homes or cars during $2016^{1}$, which might be due to incomplete implementation of voluntary smoke-free rules. To protect the health of both parents and children, coordinated efforts are needed to target lifestyle-changing interventions in parents who smoke, in concert with comprehensive tobacco prevention and control efforts aimed at reducing the availability, accessibility, and affordability of tobacco products. The American Pediatric Association's Clinical Practice Policy recommends that pediatricians address parent/caregiver tobacco dependence as part of pediatric healthcare ${ }^{18}$. Child wellness visits could be used as opportunities to screen for SHS exposure in the home, and provide parents with education on dangers of SHS exposure and with assistance on quitting tobacco smoking.

Parents of infants/toddlers were more likely to implement home and car rules than parents of teens aged 14-17 years. The adverse health effects of SHS cut across the entire span of childhood and adolescence. Hence, protection of children of all ages is critical. Adoption of smoke-free home and car rules benefits not only children in the household, but also parents themselves ${ }^{19}$. Our results show that parents that adopted complete smoke-free home rules were less likely to initiate smoking if they have never started, as well as more likely to attempt to quit or quit successfully. Adoption of comprehensive smoke-free policies at the state and local level might encourage voluntary adoption of car and home smoke-free rules ${ }^{20}$.

While prevalence of any combustible tobacco use observed among US parents in this study was slightly lower than that of US adults overall (14.3\% vs $15.1 \%$ among the general population in 2015) ${ }^{21}$, patterns of tobacco use disparities among parents were consistent with those observed among all US adults overall ${ }^{21}$. Tobacco use prevalence in our study was disproportionately higher among parents who had lower education level, annual household income of $<20 \mathrm{~K}$, veterans, living in nonmetropolitan areas, and in single-parent families. Disparities were also seen in adoption of smoke-free home and car rules. For example, non-Hispanic Black parents had the lowest prevalence of adoption of home smoke-free rules. This agrees with previous research documenting higher prevalence of SHS among non-Hispanic Black youth ${ }^{22}$. Intensified implementation of population-based interventions including increasing tobacco product prices, implementing and enforcing comprehensive smoke-free laws, and warning about the dangers of tobacco use can help reduce tobacco use $^{23}$. Several public health campaigns are targeted at parents to help them quit. For example, since 2012, CDC's Tips From Former Smokers' Campaign has educated the public about the dangers of SHS, including asthma attacks among children of smokers triggered by SHS exposure ${ }^{24}$.

\section{Strengths and limitations}

The strength of this study is the use of a nationally representative dataset of US parents to measure use of a diverse range of tobacco products. Furthermore, the ability to generate sub-national estimates with TUS-CPS provides implicative data which can inform policy and practice tailored to specific populations. Nonetheless, this study has some limitations. First, parental smoking behavior might be under-reported due to social desirability bias. Furthermore, adoption of smoke-free home rules might be over-reported in TUS-CPS because of social desirability bias and potential differences in perceptions about 'smokefree' rules. A previous qualitative study indicates that some people who smoke on an indoor balcony or out the window do not consider themselves as smoking inside ${ }^{25}$. Second, the cross-sectional nature of the survey does not allow us to establish causal relationships between the presence of smoke-free rules and smoking-related outcomes. Third, we could not assess the number of smokers in the household since parents reported only their tobacco use status, and not of their spouses, partners, grown up children, or other household members. The number of children living with a smoker might be underestimated if the surveyed parent is a non-smoker, but the other parent or household member(s) is. Similarly, the totals include never married children living away from home in college dormitories, in which case, the number of children living with the surveyed parent might be overestimated. Finally, these estimates do not include parents or their children who are in the military, civilians stationed overseas, or those in other institutionalized settings.

\section{CONCLUSIONS}

One in four US parents used a tobacco product, and 11.04 million children lived with a parent that used any form of tobacco. Overall, $91.3 \%$ of parents reported completely prohibiting smoking in their home; smoke-free home rules had a beneficial effect on parents in terms of preventing smoking initiation among never smokers, encouraging quit attempts among current smokers, and promoting sustained quitting among former smokers. Adoption of voluntary 
home and car rules, in concert with implementation of comprehensive tobacco-free laws and consultation to educate parents on the dangers of tobacco use around children can reduce SHS exposure among US youth.

\section{REFERENCES}

1. Child Trends DataBank. ChildTrends. Accessed July 7, 2021. https://www.childtrends.org/indicators?a-z\#_edn10

2. Tong VT, Dietz PM, Morrow B, et al. Trends in Smoking Before, During, and After Pregnancy - Pregnancy Risk Assessment Monitoring System, United States, 40 Sites, 2000-2010. MMWR Surveill Summ. 2013;62(6):1-19. Accessed July 7, 2021. https://www.cdc.gov/mmwr/pdf/ ss/ss6206.pdf

3. U.S. Department of Health and Human Services. The Health Consequences of Smoking-50 Years of Progress: A Report of the Surgeon General. 2014. Accessed July 7, 2021. https:// www.ncbi.nlm.nih.gov/books/NBK179276/pdf/Bookshelf_ NBK179276.pdf

4. Collins BN, Wileyto EP, Murphy MF, Munafò MR. Adolescent Environmental Tobacco Smoke Exposure Predicts Academic Achievement Test Failure. J Adolesc Health. 2007;41(4):363370. doi:10.1016/j.jadohealth.2007.04.010

5. Ho SY, Lai HK, Wang MP, Lam TH. Exposure to secondhand smoke and academic performance in non-smoking adolescents. J Pediatr. 2010;157(6):1012-1017.e1. doi:10.1016/j.jpeds.2010.06.013

6. Filippidis FT, Agaku IT, Vardavas CI. The association between peer, parental influence and tobacco product features and earlier age of onset of regular smoking among adults in 27 European countries. Eur J Public Health. 2015;25(5):814818. doi:10.1093/eurpub/ckv068

7. Agaku IT, Perks SN, Odani S, Glover-Kudon R. Associations between public e-cigarette use and tobacco-related social norms among youth. Tob Control. 2020;29(3):332-340. doi:10.1136/tobaccocontrol-2018-054728

8. Homa DM, Neff LJ, King BA, et al. Vital Signs: Disparities in Nonsmokers' Exposure to Secondhand Smoke - United States, 1999-2012. MMWR Morb Mortal Wkly Rep. 2015;64(4):103-108. Accessed July 7, 2021. https://www. cdc.gov/mmwr/pdf/wk/mm6404.pdf

9. Alboksmaty A, Agaku IT, Odani S, Filippidis FT. Prevalence and determinants of cigarette smoking relapse among US adult smokers: a longitudinal study. BMJ Open. 2019;9(11):e031676. doi:10.1136/bmjopen-2019-031676

10. King BA, Babb SD, Tynan MA, Gerzoff RB. National and State Estimates of Secondhand Smoke Infiltration Among U.S. Multiunit Housing Residents. Nicotine Tob Res. 2013;15(7):1316-1321. doi:10.1093/ntr/nts254

11. Centers for Disease Control and Prevention. State System Vehicles Fact Sheet. Centers for Disease Control and Prevention; 2021. Updated June 11, 2021. Accessed July 7, 2021. https://www.cdc.gov/statesystem/factsheets/ vehicles/Vehicles.html

12. Office of the Assistant Secretary for Public and Indian
Housing, U.S. Department of Housing and Urban Development. Instituting Smoke-Free Public Housing. Office of the Assistant Secretary for Public and Indian Housing, U.S. Department of Housing and Urban Development; 2016. Docket No. FR 5597-F-03. December 5, 2016. Accessed July 7, 2021. https://www.govinfo.gov/content/pkg/FR-201612-05/pdf/2016-28986.pdf

13. Tynan MA, Holmes CB, Promoff G, Hallett C, Hopkins M, Frick B. State and Local Comprehensive Smoke-Free Laws for Worksites, Restaurants, and Bars - United States, 2015. MMWR Morb Mortal Wkly Rep. 2016;65(24):623-626. doi:10.15585/mmwr.mm6524a4

14. Walton K, Gentzke AS, Murphy-Hoefer R, Kenemer B, Neff LJ. Exposure to Secondhand Smoke in Homes and Vehicles Among US Youths, United States, 2011-2019. Prev Chronic Dis. 2020;17:E103. doi:10.5888/pcd17.200107

15. Agaku IT, Singh T, Rolle I, Olalekan AY, King BA. Prevalence and Determinants of Secondhand Smoke Exposure Among Middle and High School Students. Pediatrics. 2016;137(2):e20151985. doi:10.1542/peds.2015-1985

16. Lidón-Moyano C, Fu M, Pérez-Ortuño R, et al. Third-hand exposure at homes: Assessment using salivary cotinine. Environ Res. 2021;196:110393. doi:10.1016/j.envres.2020.110393

17. Burton A. Does the smoke ever really clear? Thirdhand smoke exposure raises new concerns. Environmental Health Perspectives. 2011;119(2):A71-A74. doi:10.1289/ehp.119-a70

18. Farber HJ, Groner J, Walley S, Nelson K; SECTION ON TOBACCO CONTROL. Protecting Children From Tobacco, Nicotine, and Tobacco Smoke. Pediatrics. 2015;136(5):e1439-e1467. doi:10.1542/peds.2015-3110

19. Kingsbury JH, Reckinger D. Clearing the Air: Smoke-Free Housing Policies, Smoking, and Secondhand Smoke Exposure Among Affordable Housing Residents in Minnesota, 20142015. Prev Chronic Dis. 2016;13:E111. doi:10.5888/ pcd13.160195

20. Passey ME, Longman JM, Robinson J, Wiggers J, Jones LL. Smoke-free homes: what are the barriers, motivators and enablers? A qualitative systematic review and thematic synthesis. BMJ Open. 2016;6(3):e010260. doi:10.1136/bmjopen-2015-010260

21. Jamal A, King BA, Neff LJ, Whitmill J, Babb SD, Graffunder CM. Current Cigarette Smoking Among Adults - United States, 2005-2015. MMWR Morb Mortal Wkly Rep. 2016;65(44):1205-1211. doi:10.15585/mmwr.mm6544a2

22. Brody DJ, Lu Z, Tsai J. Secondhand Smoke Exposure Among Nonsmoking Youth: United States, 2013-2016. U.S. Department of Health and Human Services, Centers for Disease Control and Prevention, National Center for Health Statistics; 2019. NCHS Data Brief, no 348. August 2019. Accessed July 7, 2021. https://www.cdc.gov/nchs/data/ databriefs/db348-h.pdf

23. World Health Organization. Progress report on technical matters related to Articles 4.2.d (gender issues), 5.3 (tobacco 
industry interference), 17 and 18 (alternative livelihoods and protection of the environment) and 19 (liability). World Health Organization; 2018. Accessed July 7, 2021. https:// www.who.int/fctc/cop/sessions/cop8/FCTC_COP_8_9-EN. pdf?ua $=1$

24. Murphy-Hoefer R, Davis KC, King BA, Beistle D, Rodes R, Graffunder C. Association Between the Tips From Former Smokers Campaign and Smoking Cessation Among Adults, United States, 2012-2018. Prev Chronic Dis. 2020;17:E97. doi:10.5888/pcd17.200052

25. Rosen LJ, Lev E, Guttman N, et al. Parental Perceptions and Misconceptions of Child Tobacco Smoke Exposure. Nicotine Tob Res. 2018;20(11):1369-1377. doi:10.1093/ntr/ntx169

\section{CONFLICTS OF INTEREST}

The authors have completed and submitted the ICMJE Form for Disclosure of Potential Conflicts of Interest and none was reported.

\section{FUNDING}

There was no source of funding for this research.

ETHICAL APPROVAL AND INFORMED CONSENT

Ethical approval and informed consent were not required in this study which used existing data from a national survey.

DATA AVAILABILITY

The data supporting this research is available from the following sources:
DOI: https://cancercontrol.cancer.gov/brp/tcrb/tuscps/ questionnaires-data

\section{AUTHORS' CONTRIBUTIONS}

ITA conceptualized and designed the study and drafted the initial manuscript. NK, SO and TNT helped conceptualize the study, assisted in the statistical analyses, and critically reviewed and revised the manuscript.

All authors approved the final manuscript and agreed to be accountable for all aspects of the work.

PROVENANCE AND PEER REVIEW

Not commissioned; externally peer reviewed. 